\title{
L'invention d'une politique publique : un patrimoine urbaniste à Nantes ?
}

The invention of a public policy: a heritage planner in Nantes?

Laurent Devisme and Amélie Nicolas

\section{(2) OpenEdition \\ Journals}

Electronic version

URL: http://journals.openedition.org/rge/5067

DOI: $10.4000 /$ rge.5067

ISSN: 2108-6478

Publisher

Association des géographes de l'Est

Printed version

Date of publication: 30 December 2013

ISSN: 0035-3213

\section{Electronic reference}

Laurent Devisme and Amélie Nicolas, «L'invention d'une politique publique : un patrimoine urbaniste à Nantes? », Revue Géographique de l'Est [Online], vol. 53 / 3-4 | 2013, Online since 02 July 2014, connection on 08 September 2020. URL : http://journals.openedition.org/rge/5067 ; DOI : https:// doi.org/10.4000/rge.5067

This text was automatically generated on 8 September 2020

Tous droits réservés 


\section{L'invention d'une politique publique : un patrimoine urbaniste à Nantes?}

The invention of a public policy: a heritage planner in Nantes?

Laurent Devisme and Amélie Nicolas

\section{Introduction}

1 Le patrimoine, pris dans une acception large, semble être devenu un véritable instrument au service de l'action publique. Déjà F. Choay pouvait-elle souligner dans son essai sur l'allégorie patrimoniale une tendance au tout-patrimoine posant en miroir la question des difficultés des sociétés à se projeter et à faire des choix (Choay, 1992). Désormais, dans bien des collectivités locales en France, l'argument patrimonial se loge de plus en plus dans l'action urbaine. A Nantes, la réponse politique de choisir un adjoint au patrimoine et de créer une nouvelle Direction du Patrimoine et de l'Archéologie au début du quatrième mandat de Jean-Marc Ayrault en 2008, est significative, d'autant que Nantes n'a jamais été considérée, du moins par les acteurs politiques locaux, comme une ville patrimoniale, à la différence de Lyon ou Bordeaux par exemple.

2 Au moment d'amorce d'institutionnalisation d'une nouvelle politique locale, il s'agit ici d'engager une réflexion sur la montée à l'agenda politique des questions patrimoniales à Nantes, interrogeant alors la manière dont ces questions sont qualifiées ou requalifiées dans le champ de l'action publique urbaine. Si l'analyse des politiques publiques conduit souvent à comprendre comment se joue un travail de régulation des relations par le consensus et l'idée force d'une « culture partagée » (Boussaguet, Jaquot, Ravinet, 2010), il est proposé ici, en décryptant les enjeux divers touchant à l'urbanisme et au projet urbain d'une part et au patrimoine d'autre part, d'informer la nature de ces relations, depuis les services qui les pilotent. Ces services entrent en outre sur la même période dans une nouvelle phase, celle du passage à l'échelle communautaire 
(urbanisme) et de la mutualisation d'un certain nombre de compétences, le patrimoine et l'archéologie restant, eux, une prérogative municipale. Comment différentes dimensions (urbanisme, patrimoine, culture, tourisme) de l'action urbaine se côtoientelles? Quels rôles jouent différentes expertises, commissions, conseils scientifiques dans un repositionnement des cultures professionnelles ? Le mouvement qui pousse les acteurs du patrimoine à chercher à rendre davantage opérationnelles leurs expertises et leurs prescriptions est particulièrement intéressant (section 1). Dans le même temps, les urbanistes amenés à prôner la densification par temps de ville renouvelée sur ellemême rencontrent plus souvent qu'avant des contre-arguments alors qu'ils s'estiment légitimes sur la place (section 2). Après la mise au miroir de ces cultures professionnelles, il est intéressant de préciser les promesses d'une approche de l'action publique urbaine travaillant au plus près de ces matrices tout en choisissant une visée «micrologique » qui privilégie des entrées par l'espace et par ce que les ajustements réciproques des acteurs produisent à l'échelle de "dossiers spatiaux » particuliers (section 3).

\section{Les enseignements de la création d'une nouvelle direction municipale}

3 Le design institutionnel connaît de fortes transformations depuis la décentralisation en France. Les moments de création et de mise en place d'un nouveau service et a fortiori d'une nouvelle direction sont certes révélateurs d'évolutions concernant les pouvoirs urbains mais il faut ici regarder les épreuves qu'ils rencontrent pour assoir leur autorité. Dans le cas présent, c'est aussi bien la légitimité scientifique qu'urbaine qui est en jeu dans le rodage institutionnel de la nouvelle direction du patrimoine et de l'archéologie à Nantes (DPARC).

4 Les temporalités de l'urbanisme et du patrimoine sont a priori contradictoires. Un des enjeux de la constitution de la DPARC est bien d'articuler ces temporalités, celle de l'instruction patrimoniale s'appuyant sur l'outil principal de l'inventaire du patrimoine et celle de la fabrication du projet architectural ou urbain, s'appuyant elle sur un ensemble d'études préalables et de pratiques spécifiques liées à la culture du projet architectural. Cette articulation pose l'enjeu, depuis la DPARC, d'une mise à l'épreuve de cultures professionnelles relevant ordinairement du champ de la culture alors qu'elle tente une entrée en urbanisme où elle doit construire sa légitimité.

\section{A. Enjeux d'une nouvelle Direction : de l'ambivalence des missions}

Stéphane Junique, figure totalement hors du sérail des milieux du patrimoine local ${ }^{1}$, est devenu le18e adjoint au Maire de Nantes attaché à la question du patrimoine et de l'archéologie en 2008. Unensemble de polémiques locales autour des enjeux de considération du patrimoine et principalement de l'archéologie dans le déroulé des projets urbains contemporains avait pu motiver cette réaction politique. La plus saillante d'entre elles, celle de l'îlot Lambert, a vu la Ville accusée, en pleine campagne électorale, d'ignorer des fouilles archéologiques dans le quartier historique et secteur sauvegardé de Nantes et ainsi de plier aux pressions des promoteurs immobiliers, alors même que la responsabilité en matière d'archéologie préventive incombe ordinairement à l'Etat via l'Institut National de Recherche en Archéologie Préventive 
(INRAP). Autre saillance, celle survenue à l'occasion de la décision du port autonome de démonter une grue (la grise) sur le domaine portuaire qui, à l'instar d'une autre grue (la jaune) commençait à faire repère pour les nantais. In extremis, avec la mobilisation d'anciens de la Navale notamment, cette grue a été cédée pour l'euro symbolique à la Ville de Nantes qui l'a restaurée et inscrite dans le skyline postindustriel de la pointe Ouest de l'île de Nantes. Cette montée à l'agenda politique de la question patrimoniale prend ainsi corps au contact d'acteurs sociaux sachant jouer sur le caractère émotionnel du débat, composant avec les médias locaux et provoquant alors l'élargissement du public concerné à des controverses ne touchant initialement qu'un nombre limité de personnes (Cobb, Elder, 1983 ; Cefai, Terzi, 2012).

La DPARC, créée en 2008, dépend bien de la Direction générale de la Culture mais vient confirmer cependant la volonté des élus locaux de ne plus réduire les questions patrimoniales et archéologiques à un faible maillon de la politique culturelle municipale. La Ville s'appuyait auparavant sur une seule fonction de médiation patrimoniale dans le cadre d'un emploi d'animateur du patrimoine obtenu en 2002 en même temps que le label Ville d'Art et d'Histoire, et sur un élu, conseiller municipal peu actif.

7 Le Plan Patrimoine sur lequel s'organise la Direction engage l'élu et le service à considérer désormais, dans le cadre de la mise en place d'une nouvelle politique publique patrimoniale, que leurs prérogatives relèvent bien du champ de l'urbanisme. "La question du patrimoine, rappelait Stéphane Junique, n'est pas seulement une approche culture, c'est aussi une approche urbaine, la question de l'aménagement, c'est la problématique du bâti, c'est la problématique de l'urbanisme au sens architectural du terme, mais c'est aussi l'attractivité, comment le patrimoine participe à la logique de rayonnement de la ville. Donc on le voit bien, on est sur des approches qui sont très morcelées jusqu'à présent. Et tout l'intérêt, notamment de ce mandat, c'est de faire qu'il y ait du lien entre ces différentes problématiques. Et la Direction du patrimoine doit jouer ce rôle » (entretien avec S. Junique, 2008). L'injonction politique est double cependant: d'un côté, le patrimoine doit faire son entrée en urbanisme, et de l'autre, il doit garder sa spécificité, plus traditionnelle, de service culturel, auquel renvoie d'ailleurs la nomination à la tête de la DPARC, d'une directrice, conservatrice en chef des monuments historiques avec une spécialité "musée », qui a longtemps dirigé le Château des Ducs de Bretagne, et qui rappelle là l'ancrage historique des métiers du patrimoine en France.

La nouvelle Direction qui porte la politique publique en construction articule ainsi des missions qui restent floues et qui ne sont pas sans obstacles : d'une part elle est engagée à prendre un rôle qui pourrait relever d'une aide à la maîtrise d'ouvrage publique sur des opérations d'aménagement urbain et c'est donc là un lent processus de conquête de prérogatives pour une Direction du Patrimoine, portée politiquement mais pas ou très peu installée dans les rouages administratifs de l'action publique; d'autre part, elle suit des missions de médiation en rapport avec des acteurs externes légitimes qui ont pu "monter au créneau », des "lanceurs d'alerte " (Chateauraynaud, Torny, 1999) qui peuvent être des universitaires, des membres d'association, des habitants. C'est probablement (cf. infra, sous point C) le détricotage d'une association dotée de nombreuses attributions (Nantes Renaissance) qui explique un certain nombre d'interventions publiques visant à sensibiliser à des enjeux patrimoniaux. Aujourd'hui, l'association Forum Nantes Patrimoine joue un tel rôle de vigie qui peut aussi bien être 
un appui pour telle fraction de l'institution qu'une gêne si elle met la municipalité en porte-à-faux ou face à ses contradictions.

\section{B. Entrer en urbanisme. Une Direction du patrimoine mal outillée}

9 Les services d'urbanisme d'une part et les services du patrimoine d'autre part se trouvent dans une situation d'injonctions contradictoires, confirmant des représentations tenaces opposant souvent les «Patrimoniaux» aux «Bulldozers". L'appel à la ville compacte, confirmée dans l'étendard du SCOT, dans le PADD du PLU comme dans leurs traductions réglementaires, occupe de son côté une Direction de l'urbanisme prise en tenaille entre des promoteurs immobiliers, des enjeux de densification et de rentabilité foncière et des injonctions de conservation, protection et valorisation portés par la nouvelle Direction du Patrimoine.

le PLU représente bien l'argument d'objectivité réglementaire pour les urbanistes qui disposent ainsi de périmètres d'action bien précis, la DPARC peine de son côté à trouver un socle sur lequel asseoir sa légitimité et par conséquent réaliser les impératifs de son Plan Patrimoine.

11 Côté patrimoine, l'inventaire reste un instrument privilégié à même d'assurer la caution scientifique de l'argument patrimonial. La démarche de recensement, d'étude, de construction typologique et d'explication historique, reste en effet, dans les métiers du patrimoine, un gage de professionnalisme. Rappelons cette critique à l'encontre d'un « inventaire sensible » élaboré par Alexandre Chemetoff initiant le projet de l'île de Nantes, critique d'un pseudo-parti patrimonial de l'urbaniste n'ayant rien à voir avec une démarche historienne de connaissance du territoire.

12 Un travail systématique d'inventaire n'a cependant jamais été porté politiquement par la Ville, renvoyant là aux explications courantes d'une ville complexée sur sa qualité patrimoniale, qui ne s'est jamais perçue jusque récemment comme touristique ou simplement digne d'intérêt touristique. Ajoutons aussi que le souci de sauver l'économie industrielle et portuaire a pris le dessus pendant longtemps. L'inventaire général de la ville de Nantes à partir duquel pourraient travailler les agents du service est ainsi absent, plutôt, il est fractionné et inexploitable en l'état. "On est prisonniers, structurellement, de ce manque » confie la Directrice du Patrimoine et de l'Archéologie alors que la Direction ne peut s'appuyer aujourd'hui que sur une compilation de savoirs distincts qui fait allure d'inventaire mais qui n'a jamais été formalisée en un inventaire scientifique. Deux formes de production de connaissance sur la ville de Nantes ont pu être engagées : un inventaire partiel porté par deux chercheurs relevant d'un contrat entre la Ville et la DRAC; des missions d'inventaire à l'occasion des modifications ou révisions des POS puis PLU.

Dès 1988, la Ville, via l'association Nantes Renaissance qui avait en gestion les politiques de ravalement, émet le souhait de systématiser une démarche d'inventaire, principalement sur le secteur sauvegardé de Nantes. Une convention Ville-Etat s'initie alors et se traduit par l'allocation d'une subvention de la Ville à l'association pour le Développement de l'Inventaire général en Pays de Loire permettant d'engager deux chercheurs à mi-temps. Cette première démarche d'inventaire suit les campagnes de ravalement, mais avorte progressivement au motif que les données produites sont difficilement appropriables. Les chercheurs produisent en effet des articles scientifiques ou des ouvrages qui s'appuient sur une lecture d'ensembles urbains et 
architecturaux, qui sont, selon les élus et professionnels, peu opérationnels alors qu'ils pouvaient attendre une production par fiches de références à l'échelle du bâtiment. La subvention n'est pas non plus reconduite alors qu'elle engageait des fonds pris sur le budget de la Direction de l'urbanisme de la Ville en direction d'un service culturel de l'Etat ; ici encore les cultures professionnelles entre urbanisme et culture, au-delà des divisions entre Ville et Etat, ont fortement joué.

En parallèle, la Ville de Nantes a lancé des missions d'inventaire des bâtiments à protéger dans le cadre de la production ou de la révision des documents d'urbanisme. En 1980, sous le mandat du maire A. Chénard (municipalité de gauche), une mission est lancée en vue de l'élaboration du POS de 1981. Trois chercheurs, historiens de l'architecture et architectes, répondent à cette commande en engageant une démarche d'inventaire se fondant sur le repérage et la proposition de protection de tout ce qui relevait, dans la ville-centre et hors du secteur sauvegardé alors en régime spécial, d' «urbanisme volontaire». En ressortira une liste informée d'alignements, se traduisant par des «tâches noires » inscrite au POS Centre. En 1986, une telle démarche est reconduite sous la municipalité M. Chauty (municipalité de droite), avec des motivations concernant le patrimoine qui s'avèrent bien différentes de cette première expérience, le POS de 1987 étant aujourd'hui communément nommé le «POS dérégulation ». S'il est bien important de repérer ce qui mérite d'être conservé, l'enjeu de production de la ville dans cette décennie reste d'activer les constructions neuves. Cette seconde étude, finalement peu suivie alors que les élus finissent par faire euxmêmes la sélection, verra les fameuses "tâches noires" du POS devenir des simples " étoiles ». En 1993, sous le premier mandat de J-M. Ayrault, est mis sur pied le premier POS à l'échelle de la Ville de Nantes, le POS centre étant compris dans une zone Ua plus large ; à cette occasion, il est demandé aux mêmes chercheurs d'élargir un inventaire à l'échelle cette zone. Le Maire souhaite aussi étendre cette démarche aux zones Ub et une mission d'inventaire est également engagée. Parallèlement, une exposition sur l'histoire de la réglementation urbaine à Nantes est organisée par la Ville, en lien avec l'ARDEPA (association régionale pour la diffusion et la promotion de l'architecture) et pensée comme manifestation culturelle dans le cadre de la concertation engagée pour la révision en cours du plan d'occupation des sols (exposition intitulée P.O.S. SCRIPTUM, $\mathrm{Du}$ texte à la forme. La ville construite, Nantes, 16e-20es.). Plus de mille éléments du patrimoine nantais sont ainsi recensés, portant une interdiction de démolir sur avis simple de l'Architecte des Bâtiments de France. En 2007, le Plan Local d'Urbanisme est une nouvelle fois révisé donnant lieu à une inscription de 600 nouveaux éléments patrimoniaux, complétant la liste initiale ; cette nouvelle inscription permettra d'aller plus loin dans les identification du patrimoine à protéger, intégrant, au-delà des éléments construits remarquables du patrimoine nantais, les séquences urbaines ainsi que le «petit patrimoine» (puits, lavoirs, fontaines, statues, murs...). En 2012, une protection de 150 nouveaux éléments dans le cadre d'une modification du PLU est en cours d'instruction, à la demande explicite du cabinet du Maire. Cette demande, visant à renforcer l'axe fondateur du PADD "Qualité de ville à conforter dans tous les quartiers", est surtout motivée par l'enjeu de garder une vigilance sur les grands boulevards urbains soumis à des mutations urbaines importantes échappant souvent aux pouvoirs publics et faisant l'objet de nombreuses contestations habitantes. L'instruction de cette nouvelle liste est confiée aux services de l'Urbanisme en charge de la révision du PLU en association avec l'Instructeur du Droit des Sols, à même de 
faire remonter les remarques des riverains sur le terrain, et avec l'architecte conseil de la DPARC.

15 Aujourd'hui, cette absence d'un inventaire systématique du patrimoine explique largement l'enjeu de la DPARC de constituer un Système d'Information Géographique pour le patrimoine et l'archéologie à Nantes. Un tel outil de spatialisation de la connaissance patrimoniale permettrait sans doute de construire un point d'accroche solide à la Direction lui permettant de s'ajuster aux enjeux et méthodes de travail des urbanistes. A ce titre, la ville de Toulouse qui a mis en ligne un système d'information géographique «Urban-Hist » fait figure de modèle en la matière. Cet outil de gestion, de connaissance et de valorisation du patrimoine toulousain rend accessible aux services municipaux et intercommunaux, grâce à un site internet cartographique, les données de l'Inventaire, des Archives municipales et de l'Archéologie²

16 A l'absence d'un socle solide et opérationnel de connaissances du patrimoine pouvant permettre à la nouvelle Direction d' " entrer en urbanisme " sur la base d'une relative autonomie et d'un pouvoir d'interpellation, s'ajoute une posture des professionnels du patrimoine qui remet facilement en cause l'action des urbanistes désormais que les champs d'implication du patrimoine se sont élargis à la ville. Il y a bien une rupture entre les cultures professionnelles des services de l'urbanisme et du service du patrimoine, source de tensions. Cette rupture est également confirmée par le rôle délicat d'instruction et de gestion de controverses ou d'affaires patrimoniales, souvent initiées par la société civile et qui ressort plutôt d'un service culturel que d'un service d'urbanisme.

\section{C. «La carpe, le lapin, la chèvre et le choux ». La nécessité de « faire avec » les vieilles prérogatives}

Parce que Direction rattachée à la Culture, prérogative de la Ville de Nantes, la DPARC reste dans une position assez inconfortable qui renforce des obstacles à une entrée en urbanisme. Selon un chargé de mission de la DGDU, "c'est assez complexe, être à la fois dans l'urbanisme et le culturel, ménager la carpe, le lapin, la chèvre, le choux. " (entretien avec un chargé de mission). Il a bien été fait le choix de ne pas rattacher la nouvelle Direction aux services de l'urbanisme, confirmant la distorsion entre les "patrimoniaux » d'une part et les «bulldozers » d'autre part. Pour les agents de la DPARC, ce rattachement renforce cependant l'enjeu de scientificité de l'instruction patrimoniale qui se démarque ainsi des approches techniciennes des services d'urbanisme. Dans le contexte actuel de montée en puissance de la métropole, les agents municipaux de la DPARC peuvent se départir de leur absorption dans un «régime technocratique » et arguer d'appartenir encore au champ municipal de la culture, confirmant la volonté politique mais aussi la reconnaissance d'une démarche scientifique possible et particulière pour le patrimoine.

Plus nécessairement, il s'agit bien de composer avec les notabilités locales et pour la nouvelle Direction de "faire avec» de vieilles prérogatives, celles de suivre les revendications patrimoniales, instruites par les sociétés d'histoire locale, ou par des « lanceurs d'alerte » dont l'argument patrimonial vient justifier la prise de position. Ce n'est pas une jeune affaire à Nantes. La mise en place par la municipalité, en 1985, de l'association Nantes Renaissance, réunissant alors les membres de la société civile, l'Architecte des Bâtiments de France et des agents municipaux en détachement peut 
déjà être considérée comme la proto-histoire d'une politique publique patrimoniale à l'initiative de la Ville à Nantes. Aux côtés d'actions de sensibilisation et de médiation, l'association se voit confier une mission générale pour « explorer, susciter et guider des actions de réhabilitation dans le centre ancien » (document de communication). Elle suit principalement les campagnes de ravalement de la ville et travaille de concert avec les services de l'urbanisme dans le cadre des OPAH. Face à l'importante latitude, notamment budgétaire, dont bénéficiait l'association, et surtout par le pouvoir qu'elle pouvait prendre via des donneurs d'ordre issus de la société locale, la Ville a ensuite cherché à mettre un terme à cette situation qui lui voyait imposer un véritable lobby. La création de la DPARC, réintégrant en son sein le personnel municipal détaché à Nantes Renaissance, marque la fin de cette hégémonie associative. L'association n'est cependant pas dissoute et les adhérents continuent leur activité en direction de la sauvegarde, de la restauration et de la transmission du patrimoine à Nantes. Autre association ayant pris de l'ampleur, le Forum Nantes Patrimoine réunissant surtout des érudits locaux très en alerte sur les transformations de la ville et à même d'initier et d'instruire d'importantes controverses patrimoniales. La DPARC est ainsi dans une position très ambivalente : l'enjeu de connaissance qu'elle escompte et qui légitime sa position, son rôle et son expertise dans le champ institutionnel reste largement alimenté, à défaut d'un inventaire systématisé, par les productions de ces érudits locaux du patrimoine. La mise en place d'un Conseil nantais du Patrimoine et de ses commissions scientifiques accueillant certains membres de ces associations sont bien le marqueur de cette inconfortable position de la Direction. Ces commissions sont un véritable outil de validité scientifique des propositions de la DPARC mais l'engage en retour à la gestion de personnalités, souvent contestataires, qui accentue alors l'image d'une Direction conservatrice et freinant la conception urbaine. L'enjeu pour la DPARC reste bien de contenir la prolifération patrimoniale et la prise de pouvoir sur la ville des érudits locaux alors même qu'ils appuient la connaissance scientifique du patrimoine dont elle a besoin.

Cette proximité de la DPARC avec les services culturels de la Ville de Nantes l'amène également dans un conflit de légitimité avec une conception touristique du patrimoine qui s'affiche à Nantes, dans le cadre de la toute nouvelle Société Publique Locale « Le Voyage à Nantes ${ }^{3}$. Le "VAN" souligne un enjeu fort des relations entre politiques culturelles, politiques touristiques et politiques d'internationalisation de l'image de la ville. La DPARC, par les missions de médiation qu'elle porte, se trouve en concurrence avec cette représentation de la ville qui avait amené par exemple, à l'été 2012, à la mise en place par le Voyage à Nantes d'un parcours touristique en ville avec lequel la DPARC a dû un minimum composer bien que les conceptions de la mise en valeur des espaces urbains soient différentes de part et d'autre.

Dans les trente dernières années, l'entrée du patrimoine en urbanisme a connu différentes figures qu'il est utile de résumer en gardant en tête une évolution respective des cultures professionnelles. Si les professionnels du patrimoine ont progressivement quitté une représentation réduite à l'objet pour aller vers des ensembles cohérents (c'était certes déjà le cas des secteurs sauvegardés instaurés en 1962 mais cela s'accentue dans les années 1990, dont témoigne le nombre de ZPPAUP, désormais AVAP), les urbanistes ont également de leur côté investi la ville existante en reconnaissant ses qualités et en recourant à une réflexion opérationnelle sur l'espace public (cf. infra). 


\section{L'histoire parallèle d'un service urbanisme qui « fait déjà patrimoine »}

21 L'héritage institutionnel revient à des partitions nettes. Monuments historiques classés et inscrits, architectes des bâtiments de France, secteurs sauvegardés, archéologues sont tous des actants traditionnellement mis en mouvement par l'Etat, dans une conception supra-territoriale et un intérêt qui est à la fois de connaissance et esthétique. Assez loin de là, les services d'urbanisme qui mettent en musique le développement de la ville, composés de cultures d'ingénieurs et d'architectes principalement.

\section{A. Le sens du patrimoine chez les architectes-urbanistes}

Côté urbanisme, on entend plutôt que l'« on fait déjà patrimoine »: aussi bien les études préalables que l'évolution d'un service vers la qualité et l'espace public en témoignent dans le cadre de ce que l'on peut appeler un urbanisme culturaliste. D'où une réception initiale mitigée de l'arrivée de la DPARC par la DGDU (Direction Générale du Développement Urbain) : incompréhension initiale, remise en question du métier et de la conduite de missions classique de l'urbaniste qui, la plupart du temps architecte de formation, est loin d'être insensible à l'épaisseur historique de la ville (deux acteurs nous ont rappelé leurs enjeux de diplôme d'architecte d'abord orientés vers l'histoire urbaine). Alors que les "patrimoniaux" sont ordinairement perçus comme hors des services, "l'ennemi est dedans" (un chargé de mission) avec la DPARC. Il a donc fallu des appropriations respectives des usages propres au métier de l'autre!

L'entrée en urbanisme du patrimoine pose avant tout la question de la perturbation d'un champ existant. Ce champ de l'urbanisme public peut ici être considéré à deux niveaux. Le premier est celui des «préalables incontournables». Les études urbaines que commande la collectivité ne sont en effet pas dénuées d'enjeux patrimoniaux ni les études d'impact obligatoires dans le cadre de grandes opérations. Ce niveau dans lequel l'urbanisme est partie prenante de l'instruction de la question est cela dit rarement abordé comme pertinent pour des acteurs patrimoniaux. Les questions principales sont ailleurs: développement de l'attractivité, renforcement de pôles commerciaux, renouvellement d'un secteur faubourien, réalisation d'une nouvelle centralité... Les motivations de ces études sont clairement orientées vers la transformation urbaine. La question de la centralité en est un très bon analyseur (cf. infra). L'autre niveau nous semble plus important, c'est celui des cultures professionnelles en place, des manières de faire des urbanistes municipaux. Il faut ici recourir à une histoire professionnelle et organisationnelle située.

Le patrimoine dans le service urbanisme où dominent classiquement des architectes et des ingénieurs a pu connaître diverses conceptions. Jusque dans les années 1980 s'impose une conception ingénieriste de la voirie, qui se matérialise dans le traitement de la plupart des espaces publics qui sont des conduits pour unités véhiculaires rapides (exemple paradigmatique, le traitement du cours des Cinquante Otages). Par ailleurs, en période pré-électorale, dès 1987-1988, le devenir du site des chantiers de l'île de Nantes fait débat, clivant deux options, l'une développementaliste -tertiaire, l'autre mémorielle. Cette démarcation rend visible certaines associations dont Nantes la bleue 
qui défend globalement l'image portuaire et fluviale de la ville. A l'occasion de l'installation d'une municipalité de gauche en 1989, l'idée combinée d'une nouvelle ligne de tramway et d'une couture des deux centres principaux amène au premier plan une conception architecturée et urbanistique de l'espace public. La direction de l'urbanisme voit ainsi se créer une cellule Nouvelle Centralité et Tramway, structure de projet qui tranche avec les routines instructives préalables. Le projet phare du premier mandat de Jean-Marc Ayrault est bien la réfection du Cours des Cinquante Otages, nouvelle centralité nantaise augurant d'une décennie placée sous les auspices d'un espace public aménageur (et c'est dans la même période que se diffusent les expériences de Bologne, Barcelone, le travail de JM Wilmotte à Lyon...). La logique technicienne des «métiers de tuyaux et de voirie » tend clairement à être reléguée au deuxième plan. Une nouvelle vision se déploie également dans la direction des transports. A Nantes, l'ingénieur Y. Le Gal (mission Plan de Déplacements) déploie une vision de voirie partagée vue comme révolutionnaire dans les années 1990. Il est le quasi auteur des mini rond-points à l'encontre des "grands giratoires DDE ». Les acteurs de cette époque parlent volontiers d'une approche par la fonction et la qualité. L'une des forces de cette période est probablement l'implication des mêmes professionnels aussi bien dans la maîtrise d'ouvrage que dans la maîtrise d'œuvre ensuite (tramway, L2 nord). La vision d'un espace public structurant se déploie également sur les quartiers d'habitat social, dans une logique non discriminante et de développement de l'accessibilité urbaine ${ }^{4}$. Le Cours des Cinquante Otages a également occasionné l'expression de l'association Nantes la Bleue (enjeu du recreusement du lit de l'Erdre), il a certes connu une polémique à l'occasion de la suppression des platanes qui le ponctuaient mais sans conséquences majeures.

La Cellule Nouvelle Centralité et Tramway évolue ensuite vers des compétences plus larges et devient la Cellule Espaces publics avant de gagner le statut de Direction de l'Espace public (1996). A partir du deuxième mandat socialiste (1995-2001), les enjeux de projet se précisent sur l'île de Nantes; le District, désormais dirigé par L. Théry, monte en puissance du côté des études urbaines (place Sarrail à Rezé, route de Vannes sur le territoire de Saint-Herblain) et sur la grande échelle estuarienne. C'est en 1998 qu'est lancé le marché de définition de l'île de Nantes par la Ville, en accord aussi bien avec le District que l'agence d'urbanisme. On peut dire, sur cette période d'aprèsdécentralisation, que c'est la sensibilité professionnelle architecturale qui imprime une orientation nette sur l'espace public. L'activisme urbanistique tranche avec des questions patrimoniales qui tendent à se restreindre au "domaine» du secteur sauvegardé. Chasse gardée de l'ABF, ici comme dans le périmètre lié aux monuments historiques, les enjeux de développement de la ville se font plutôt ailleurs. Et lorsque l'on est en situation de frottement, à la limite d'un périmètre par exemple, on peut alors saisir les différences entre deux mondes. Anecdotique peut-être, la petite affaire de la bulle de vente installée sur le quai de la Fosse pour promouvoir un programme privé en face sur l'île de Nantes a permis un bras de fer avec l'ABF. Il n'était alors pas rare d'entendre en réunion les sarcasmes des aménageurs relatifs au vieux monde poussiéreux des défenseurs des vieilles pierres, non pour défendre l'esthétique de la bulle de vente mais plutôt le côté dérisoire d'un trouble esthétique temporaire par rapport aux enjeux de reconstruire la ville sur elle-même sur l'île de Nantes. Cela dit, pendant toute cette période, la sensibilité patrimoniale est montée inexorablement sur certains autres secteurs (notamment l'îlot Boucherie, cf. infra). 


\section{B. Une direction certes communautarisée et mutualisée mais déboussolée} de nombreuses questions de légitimité de l'appareil d'intervention publique ${ }^{5}$. C'est plus que jamais au sein des SEM que se déploie l'urbanisme de projet, en particulier au sein de la Samoa créée en 2003 pour l'aménagement de l'île de Nantes, dirigée par L. Théry, futur grand prix de l'urbanisme. DGDU, DGRU, la direction adjointe à l'urbanisme au sein de la communauté urbaine de Nantes (créée en 2001) se cherche régulièrement un nouveau directeur. Pas moins de 5 directeurs en 8 ans se succèdent sans qu'ils parviennent à faire exister leurs prérogatives. Tantôt ils sont débordés par l'activisme et le professionnalisme des SEM d'aménagement, tantôt en deuxième ligne par rapport à la ligne historique des urbanistes de la Ville de Nantes. Ces extérieurs à la place locale ${ }^{6}$ n'ont pas suffisamment d'autorité et les élus peinent à trouver un profil pertinent : un urbaniste reconnu a toutes chances d'aller au conflit avec le directeur de la Samoa, un gestionnaire sera vite perçu d'abord comme un technocrate... Finalement, et c'est un pis aller, la direction de l'urbanisme est rétrogradée en 2010 en un département du développement urbain, au sein de la Direction Générale Proximité et territoires. Les préoccupations sont pendant cette période très organisationnelles et de l'ordre de la planification : il s'agit de repenser les rapports entre la centrale et les pôles de proximité, de reconfigurer des outils de planification sur l'habitat (PLH) et les déplacements (PDU), de communautariser les ZAC.

La dimension patrimoniale, à l'échelle communautaire, s'exprime plutôt sur un registre identitaire, d'emblématisation minimale des 24 communes de l'agglomération. Tel chargé de mission, désormais à l'échelle communautaire, cherche à déployer une culture de l'espace public à cette échelle, en dehors des grands projets centraux et sur un mode relevant d'abord de la sensibilisation, tendant à l'élaboration de documents guides : charte de l'espace public par exemple. En outre, c'est plutôt un modèle de " petite grande ville » que l'on voit s'énoncer chez les élus, avec la figure récurrente du village et du petit patrimoine. La ville des courtes distances, de la mise en valeur de la proximité est alors associée à l'héritage d'une forme urbaine plus proche du hameau et du bourg parfois fantasmé, le patrimoine pouvant être support des nouvelles fonctions que l'on cherche à donner à de petits pôles ou centres, à l'échelle du quartier. C'est ici un patrimoine quasi-générique, l'identité d'un village de bord de Loire ou d'une forme typique du Sud Loire, une vasière, un petit port d'étier.

Si l'on revient vers la ville-centre qui concentre toujours largement l'expertise urbaine, les préoccupations patrimoniales au niveau de la direction de l'urbanisme de Nantes Métropole se repèrent à la fois sur un espace délimité - le site du projet de mémorial à l'abolition de l'esclavage sur le quai de la Fosse (Chérel, 2012) - il s'agit alors de révéler l'histoire de la traite négrière telle qu'on peut la lire depuis l'une des villes du négoce triangulaire et, dans la poursuite des savoir-faire urbanistiques sur l'île de Nantes, au niveau de la transformation du secteur du Bas Chantenay (cf. infra).

Retenons donc un contexte peu stabilisé pour les services d'urbanisme (incertitude sur l'échelle en œuvre, prépondérance d'acteurs-aménageurs dans des sociétés publiques locales) qui peut être aussi facilitateur pour que de nouveaux interlocuteurs puissent faire entendre leurs voix. Si le fond de plan est donc bien celui de cultures professionnelles en tension, on perçoit des signes de transformation et des modalités

Revue Géographique de l'Est, vol. 53 / 3-4 | 2013 
plus orientées vers la collaboration que le conflit, ce qu'il reste à davantage documenter.

\section{A l'épreuve du terrain, des cultures professionnelles en mouvement}

30 L'enquête menée a permis d'instruire une série de « dossiers spatiaux » qui engagent à compléter les analyses des représentations et des cultures professionnelles, rendant souvent compte des réalités des acteurs de façon assez statique, par une analyse pragmatique, permettant de voir des logiques partenariales à l'œuvre, et ainsi des tentatives de dépassement de réflexes sectoriels. Nous rentrons là dans les tentatives d'ajustements réciproques que les acteurs, dans un travail au quotidien, s'efforcent d'accomplir. Certes, pour l'heure, le rapport de force n'est pas spécialement en faveur de la DPARC mais notre attention à ce qui émerge n'en fait pas une donne déterminante.

\section{A. A la recherche d'une culture partagée...}

On peut considérer, dans les suites de 2008, que les acteurs évoqués sont dans une phase d'instruction d'un "nouveau » domaine d'action publique : c'est un temps où s'élabore, idéalement, une culture partagée. Cela se traduit notamment par la mise en place de nouvelles instances de travail, devenant un temps de négociation explicite (Gaudin, 2007) entre Direction de l'urbanisme et Direction du Patrimoine. Pour la DPARC, il s'agit de pouvoir entrer dans le circuit de décision, principalement provoquer l'instruction patrimoniale au moment même de l'instruction urbaine d'un projet, c'està-dire dans toutes les phases amont, préalables, des études urbaines. Même si très concrètement, le moment du permis de construire reste clé dans la régulation par le patrimoine, il échappe bien souvent à l'action de la DPARC, non informée ou prise de cours. La prise en compte du patrimoine, du moins des services s'attachant à son instruction, sera plus aisément permise à l'occasion de projets urbains de plus longue portée, de projets de grands territoires s'étirant dans la durée. Cette dimension procède bien d'une volonté politique, dans le fait de rendre nécessaire les études de faisabilité pour les grands projets, qui a autorisé les services du patrimoine à se départir des temporalités réglementaires très courtes d'un urbanisme du « quotidien ». Sur ce point, l'étude du quartier du Bas-Chantenay à Nantes rend compte d'une forme d'exemplarité des relations entre Direction du Patrimoine et Direction de l'urbanisme. Cette étude patrimoniale menée en collaboration avec les services de l'Inventaire est devenue une aide à la décision avec un dialogue en amont des transformations avec les services de l'urbanisme.

Du point de vue de la DPARC, il s'agissait de réussir à montrer « en quoi la connaissance patrimoniale est susceptible d'induire un projet urbain, et parfois de l'infléchir. (à montrer) en quoi la connaissance d'un territoire va (...) être un guide au projet urbain » (entretien MHJ). La valorisation du patrimoine industriel s'avancerait alors comme une propédeutique à une action concertée entre urbanisme et patrimoine. A Nantes, c'est clairement autour $\mathrm{du}$ patrimoine industriel que cette articulation est possible, elle s'est éprouvée notamment sur l'île de Nantes, en amont même du travail urbanistique et paysager d'A. Chemetoff (Nicolas, 2009 ; Devisme et al, 2006). Une autre hypothèse n'est pas à exclure. 
Concernant ces sites d'héritage industriel, le pouvoir local a vite compris l'enjeu d'un phasage, traduit parfois en nécessité d'un travail de deuil, avec la nécessité de ménager un temps long. Les études peuvent occuper alors cet espace-temps, contrairement à d'autres espaces pris dans des enjeux de plus court terme et sur lesquels n'existe aucun périmètre réflexif (les périmètres d'étude aujourd'hui) lié à un projet politique fort. C'est particulièrement le cas des boulevards et de leur densification manifeste.

En dehors du patrimoine industriel (également mis en tension via des lanceurs d'alerte comme les anciens de la Navale), les autres objets à dimension patrimoniale, présents de manière diffuse, se trouvent saisis dans des temporalités courtes liées à l'instruction des permis de construire. C'est le cas en ce qui concerne l'église de la Madeleine sur le site de l'île de Nantes, d'abord promise à la destruction et qui se trouve reconsidérée via les deux alertes d'une association et de l'ABF. Atermoiements, allongement des temporalités, obligation de réflexivité accrue pour l'aménageur, le projet de reconfiguration d'un îlot clé sur l'île de Nantes est finalement "éteint » et d'autres espaces sur l'île sont mis en avant. C'est le cas d'autres lieux vus comme des cas ou des exceptions. L'un de nos interlocuteurs, architecte-urbaniste, mobilise volontiers une grille de lecture que suggérait François Loyer concernant l'intervention patrimoniale. Trois cas de figure peuvent être identifiés, ainsi ressaisis par notre interlocuteur : «Il y a 3 catégories: ou bien vous êtes inscrit/classé et vous avez des moyens publics, ou bien vous n'êtes ni inscrit, ni classé, mais vous trouvez qu'il y a un intérêt et vous trouvez un programme, et il faudra beaucoup d'idées, de souplesse et de renouvellement (la Criée de la Rochelle, 7 ou 8 usages connus), ou bien vous n'avez rien de tout ça et c'est pas la peine d'entretenir d'illusions, vous savez pas faire, ça tombe. » (Entretien avec le Directeur de la Mission Centre-ville de la DGDU).

C'est ici que l'on voit l'importance que prend rapidement le moment réglementaire pour les politiques publiques. En effet, la prise en compte du patrimoine dans le projet est susceptible d'entraîner plusieurs altérations programmatiques: arguments des riverains qui veulent conserver l'ambiance de leur quartier et se méfient de nouveaux venus, arguments de connaissance liée à l'expertise historienne du patrimoine, arguments de contextualisation de l'opération dans une culture urbanistique qui " fait avec l'existant » et qui est traduite dans les études amont... On voit régulièrement le patrimoine être agité dans des controverses de type Nimby et ce sont les urbanistes de Nantes Métropole qui se trouvent dans la double injonction paradoxale: à la fois densifier la ville (qui reste moins dense que beaucoup d'agglomérations françaises) et en même temps contenir ou désamorcer les controverses habitantes. La Ville de SaintHerblain, deuxième en taille d'importance dans l'agglomération, connaît de son côté un nombre significatif d'oppositions à la stratégie de densification dont les professionnels de la ville-centre espèrent encore être relativement préservés ${ }^{7}$. Certes le PLU et le SCOT sont convergents mais c'est bien à l'échelle fine de l'opération, de l'îlot et de la parcelle que se négocient et s'ajustent les règles urbaines. C'est à ce niveau que les appétits des promoteurs comme la pression foncière se font les plus forts.

Dans le cadre de la dernière modification du PLU, entamée en 2012, le maire a directement passé commande d'une nouvelle inscription d'objets patrimoniaux (150). Est-ce un outil pour favoriser l'intégration de logiques patrimoniales dans les cultures professionnelles de l'urbanisme? Est-ce l'occasion d'un nouveau dialogue entre deux mondes qui ont généralement tendance à considérer avoir le monopole de la transversalité entre différents secteurs d'intervention? 


\section{B. Compromis n'est pas compromission!} auprès de l'équipe de la DPARC ont montré comment un travail d'autocritique des professionnels du patrimoine était en cours, professionnels qui semblent parfois juger des excès dont ils ont pu faire preuve par le passé. Ainsi peuvent-ils faire amende honorable pour avoir pu fustiger l'architecture contemporaine produite et ce, au-delà des prérogatives qui pouvaient être les leurs. Le projet actuel de réaménagement du passage Pommeraye, grande galerie commerciale du 19e siècle classée monument historique, contribue fortement à déplacer, à élargir, à rendre moins rigides les représentations du patrimoine et de sa conservation-valorisation dans le cadre d'un projet commercial contemporain. En effet, l'ouverture d'une seconde galerie adjacente au passage couvert historique à l'initiative d'un maître d'ouvrage privé autorise en retour, parce qu'elle la rend opportune, sa restauration. Les relations de travail s'avèrent également plus simples entre la DPARC et les chefs de projet des SEM, notamment Nantes Aménagement, qui, parce qu'ils ont des profils plus jeunes, sont en général acculturés aux problématiques spécifiques des politiques publiques, de la recherche de transversalité, d'inviter à l'action plus que de l'imposer, de fournir un cadre d'action collective et d'accompagner des processus interactifs de construction des problèmes et des solutions, d'incarner finalement cette conception du territoire comme "support de l'apprentissage de la coopération entre les acteurs" (Bonetti, Conan, Allen, 1991). Au point où la DPARC finit par promouvoir elle-même une action par projet, alors que l'interactionnisme du projet peut aussi permettre de prendre une place honorable et d'éviter la position inconfortable du face à face sectoriel.

La traduction de cette conception d'un compromis possible entre patrimoine et urbanisme a récemment pris forme au sein de la Direction de l'espace public, ayant initié une revue de projets où l'exercice de la table ronde entre $\mathrm{ABF}$, architecte de la DPARC, et maîtres d'oeuvre des espaces publics et de la modération par un architecteurbaniste de cette Direction, illustre bien le compromis opérationnel à l'œuvre dans la fabrication de la ville. Ainsi de la démolition d'un mur du parc du Grand-Blottereau annoncé par un urbaniste de la Direction des Déplacements engagé à concevoir les 1,30 mètre nécessaires au passage d'une voie vélo et prescrits par le Plan de Déplacement Urbain (PDU) et par le Plan communal des Déplacements doux (PCDD); au «non» catégorique de l'ABF, au "je préférerais ne pas» de la DPARC, s'en est suivi un « laissons le mur et aménageons une zone en amont pour informer aux cyclistes et aux conducteurs qu'ils devront, sur 30 mètres, partager la même voie » (observation d'une « revue de projet ", à la Direction de l'espace public, Nantes Métropole, octobre 2012)

De ce fait, le moment opérationnel tend à ne plus être celui exclusif de celles et ceux qui font la ville (dans le sens que l'on trouve dans le film italien de Francesco Rosi Main basse sur la ville - 1963). Plusieurs affaires montrent également des croisements entre services, des ajustements ponctuels.

L'affaire "malheureuse » des Cordeliers a montré également comment la prescription réglementaire avait pu être inefficace alors qu'un promoteur immobilier, ne suivant pas les restrictions apportées au permis de construire au moment de son accord, s'est trouvé agir en roue libre dans le secteur sauvegardé. L'évêché, propriétaire d'un ensemble architectural et patrimonial d'un ancien couvent a alors vendu à une société 
immobilière une partie de cet ensemble où étaient érigées trois chapelles fondées au XVIe siècle par des familles de négociants et notables nantais d'origine espagnole. Le promoteur ne respectant pas les prescriptions de l'ABF a abattu l'une des trois chapelles. Emerge ainsi une importante controverse initiée et suivie par l'association Forum Nantes Patrimoine pointant le problème d'une absence de contrôle des chantiers. Dans ce travail de régulation entre contraintes réglementaires posées par le PSMV du secteur sauvegardé et les projets neufs de promotion immobilière, la DPARC est amenée à jouer un rôle de conciliation opérationnelle. L'échec de cette affaire des Cordeliers a cependant son pendant «bienheureux» dans le cadre d'une opération immobilière complexe sur l'îlot Boucherie, à l'endroit même où un vieux mur d'enceinte de la ville a été retrouvé. Ici, les maîtres d'œuvre de l'opération ont engagé un travail de mise en valeur, engageant un dialogue vécu comme constructif entre acteurs du patrimoine et de l'archéologie et acteurs de l'urbanisme.

On le voit, la fabrication de la ville entraîne des arrangements qui font bouger les lignes de métiers de la ville classiquement en opposition, entre partisans de la ruine et partisans du chantier. Malgré tout, les rapports de force persistent et nous aurions tort de croire que la négociation se généralise entre des acteurs disposant de ressources identiques. Souvent, l'appel au patrimoine et à son service gestionnaire peut permettre de contrecarrer un projet immobilier qui n'aurait pas assez de crédit auprès des élus. Ainsi, le projet de restauration de la porte Saint-Pierre, porte de l'entrée est de la ville, monument suffisamment remarquable pour que personne ne remette en question sa légitimité patrimoniale, se trouve contrarié par la position de plusieurs services : la localisation de la porte sur une voie stratégique pour le passage des bus engage la Direction des Déplacements à préférer ne pas engager de travaux pour assurer la circulation; la mise en valeur de la porte impliquerait de déplacer les bornes de locations des «Bicloo », ce que la Direction des Déplacements juge impossible : enfin, la présence deux fois par an des forains sur le cours jouxtant la porte intimide les élus si des travaux de restauration de la porte et d'aménagement d'un parc archéologique aux alentours perturbaient le déroulé de la fête foraine. Ainsi, la montée à l'agenda politique du patrimoine en vue de constituer une véritable politique publique patrimoniale se heurte toujours à des résistances sectorielles qui posent la question de la légitimité même de cette politique.

\section{Conclusion}

Plusieurs " dossiers spatiaux » indiqués et documentés par les professionnels de l'urbanisme et du patrimoine à Nantes permettent de prendre la mesure de questions émergentes dans les politiques publiques spatiales. En partant d'un moment d'institutionnalisation d'un nouveau service, nous avons abordé trois moments indispensables à la mise en place d'une politique publique. L'un est instructif, c'est celui de la culture du problème et de la construction de la légitimité d'une intervention; l'autre est réglementaire et porte sur l'enjeu de codification et d'imposition de règles (le champ patrimonial est généralement essentiellement saisi par ce prisme du classement et de l'inscription) - il aurait été nettement développé si nous avions abordé l'enjeu de la révision du secteur sauvegardé ; un dernier est proprement opérationnel et tend à montrer l'importance du chantier, de l'in situ pour révéler des problèmes et amener à l'élaboration de compromis. Si nous avons surtout insisté sur le moment 
instructif de la question, c'est pour mettre en avant les frottements entre cultures professionnelles, les rapports entre expertises interne et externe et les attentes que différents acteurs placent vis-à-vis de la «fonctionnalité » patrimoniale. C'est en cherchant à « remonter le temps » (la méthode rétro-progressive qu'avait pu suggérer en son temps H. Lefebvre) que nous avons pu comprendre les fondements différents de cultures professionnelles polarisées autour de la culture savante d'un côté et de cultures professionnelles du projet urbain de l'autre. Le sens de cet écart pourrait être formulé par cette question : à qui appartient le droit de faire l'histoire de la ville?

Faisant le pari d'une possibilité de saisir les enjeux de création d'une politique publique, nous avons donc parcouru différents moments de l'institutionnalisation d'une préoccupation. Sur fond d'une histoire toujours en tension, nous avons pointé ce qui, depuis près de cinq ans, reconfigure un pragmatisme en alerte et comment, petit à petit s'invente un patrimoine urbaniste à Nantes. Via une approche ethnographique de l'action publique, à partir de récits bio-thématiques et de dossiers spatiaux, nous avons souhaité mettre en lumière à la fois une complexité et de nouveaux rapports de force. Pointons notamment des débats autour du point de vue transversal (qui le tient?), autour des rattachements institutionnels des différents domaines d'action, autour de l'évolution des tables professionnelles (cf. la pratique de la revue de projet).

\section{BIBLIOGRAPHY}

Bonetti M., Conan M., Allen B., 1991, Développement social urbain. Stratégies et méthodes, Paris, L'Harmattan

Boussaguet L., Jacquot S., Ravinet P., 2010, Dictionnaire des politiques publiques, Paris, ScPo

Bourdin A., 1991, « Pourquoi la prospective invente-t-elle des territoires ? » in Espaces et Sociétés $n^{\circ} 74-75$.

Bourdin A., 2000, La question locale, Paris, PUF

Cefai D., Terzi C. (dir.), 2012, L'expérience des problèmes publics, EHESS, Raisons Pratiques.

Chateauraynaud F., Torny D., 1999, Les sombres précurseurs. Une sociologie de l'alerte et du risque, EHESS.

Cherel E., 2012, Le mémorial de l'abolition de l'esclavage de Nantes. Enjeux et controverses (1998-2012), Rennes, PUR

Choay F., 1992, L'allégorie du patrimoine, Paris, Seuil.

Cobb R.W., Elder C.D., 1983, Participation in American Politics. The Dynamics of Agenda Building, Baltimore et Londres, The John Hopkins University Press.

Devisme L., Pasquier E., 2002, La politique de la ville par l'approche biographique. Daniel Asseray et la question urbaine. Rapport pour la DIV, LAUA, 97p. 
Devisme L. et al., 2006, L'analyse pragmatique d'un projet urbain. La mise à l'épreuve du plan-guide en projet de l'île de Nantes, rapport final de recherche pour le PUCA, programme « échelles et temporalités des projets urbains ", 215p.

Devisme L. (dir.), 2009, Nantes, petite et grande fabrique urbaine, Marseille, Parenthèses.

Gaudin J-P., 2007, Gouverner par contrat, Paris, ScPo

Mele P., 2005, « Conflits patrimoniaux et régulations urbaines », La Lettre ESO, $\mathrm{n}^{\circ} 23$, p. 51-57.

Nicolas A., 2009, Usages sociaux de la mémoire et projet d'aménagement urbain. Les héritages industriels et portuaires à l'épreuve du projet de l'île de Nantes, Thèse de Doctorat de sociologie sous la direction de Véronique Guienne. Laboratoire du CENS-EA3260. Université de Nantes.

Nicolas A., 2010, « Des espaces témoins du passé au cœur de la fabrique urbaine », Lieux Communs, n¹3, p. 166-185

Nicolas A., Zanetti T., 2013, « Patrimoine et projet urbain. Produire et valoriser la localité à Nantes, Saint-Etienne et Clermont-Ferrand ", Espaces et sociétés, numéro thématique Les aléas de la patrimonialisation urbaine coordonné par Jean-Pierre Garnier et Maria Castrillo.

\section{Liste des sigles utilisés}

ABF : Architecte des Bâtiments de France

AVAP ou AMVAP : Aire de Mise en Valeur de l'Architecture et du Patrimoine

ARDEPA : Association régionale pour la diffusion et la promotion de l'architecture

DDE : Direction Départementale de l'Equipement

DGDU : Direction Générale au Développement Urbain

DGRU : Direction Générale du Renouvellement Urbain

DPARC : Direction du Patrimoine et de l'Archéologie

DRAC : Direction Régionale des Affaires Culturelles

INRAP : Institut National de Recherche en Archéologie Préventive

OPAH : Opération Programmée d'Amélioration de l'Habitat

PLU : Plan Local d'Urbanisme

PADD : Plan d'Aménagement et de Développement Durable

PCDD : Plan Communal de Déplacement Doux

PDU : Plan de Déplacement Urbain

PLH : Plan Local de l'Habitat

POS : Plan d'Occupation des Sols

PSMV : Plan de sauvegarde et de mise en valeur (secteur sauvegardé)

SEM : Société d'Economie Mixte

SCOT : Schéma de Cohérence Territoriale

ZAC : Zone d'Aménagement Concerté

ZPPAUP : Zone de Protection du Patrimoine Architectural Urbain et Paysager 


\section{NOTES}

1. Ancien infirmier ayant repris des études à l'Ecole des Hautes Etudes en Santé publique à Rennes, responsable mutualiste et chargé d'études hospitalières et par ailleurs au parti socialiste depuis l'âge de 17 ans puis responsable de la section Nantes-Sud.

2. Rappelons que cet outil est le résultat de plus de vingt ans d'opération continue d'inventaire du patrimoine à l'initiative de la Ville de Toulouse.

3. Cette société, dirigée par Jean Blaise, figure locale s'il en est de la culture à Nantes (ancien directeur du Lieu Unique et des mythiques éditions des Allumées) regroupe en une même structure l'ancien office de tourisme, le château des Ducs de Bretagne, les cryptes de la cathédrales, le mémorial à l'abolition de l'esclavage, la manifestation Estuaire et les machines de l'île. Ce chapeautage est le signe d'une recherche manifeste de convergence entre culture et tourisme.

4. Il faut ici pointer le rôle important du premier adjoint à l'urbanisme de J-M Ayrault, D. Asseray (Devisme, Pasquier, 2002).

5. Nous renvoyons aux actes de la journée du 11 Décembre 2012, l'intercommunalité à la nantaise, MSH de Nantes, co-org L. Devisme et R. Epstein, pour un tableau plus général de caractérisation de la problématique nantaise.

6. On peut ici faire l'hypothèse qu'il n'est pas simple d'arriver à Nantes en position directoriale !

7. Cf. les actes de la séance du Pôle de recherche urbaine des Pays de la Loire du 16/11/2010 (MSH de Nantes) consacrée aux paradoxes de la densification de l'habitat : injonctions, oppositions et médiations. Disponibles en ligne: http://www.cete-ouest.developpement-durable.gouv.fr/ article.php3?id_article $=605$

\section{ABSTRACTS}

From the case of Nantes (France) which has recently developed a specific municipal office dedicated to heritage and archeology concerns with a deputy mayor to this domain, this text deals with the way how urban heritage problems transform public interventions. From the stake of professional cultures informed by the actors, we show both conflicts and articulations of visions and skills between urban and architectural heritage municipal offices and metropolitan planning offices. In relation to other participants such as associations and local historians but also to other new public structures in the domain of tourism and according to different temporalities, these offices deal with spatial issues. We suggest through their analysis a reflection on contemporary public action accounting. Assessment, rules and projects show together key moments of the relations between public services.

A partir d'un cadrage large sur Nantes et la montée à l'agenda politique de la question patrimoniale (l'arrivée d'un adjoint, la configuration d'un service "patrimoine et archéologie" au sein de la Ville), l'article interroge la manière dont les questions patrimoniales sont qualifiées dans le champ de l'action urbaine contemporaine. A partir de la fenêtre des cultures professionnelles renseignées au plus près des acteurs, il s'agit d'analyser les confrontations et articulations entre visions et savoir-faire du côté d'un nouveau service patrimonial d'une part et des services d'urbanisme d'autre part. Au contact d'autres intervenants (la société civile savante, des associations tantôt alliées tantôt défiées, une nouvelle structure de promotion touristique) et 
en fonction de temporalités tantôt longues (un projet urbain à moyen terme au programme non défini), tantôt raccourcies dans le cadre de délivrance des permis de construire, quelques « dossiers spatiaux ", où se jouent des relations complexes entre patrimoine et urbanisme, nous permettent d'instruire ces questions qui ouvrent à une réflexion sur les référentiels de l'action spatiale contemporaine. Instructions (expertises), prescriptions (règles) et opérations (chantiers) témoignent ensemble de moments clé des rapports entre services d'action publique.

Die Stadt Nantes (Frankreich) hat in jüngerer Zeit einige administrative Umwälzungen erfahren: die Schaffung des Postens eines stellvertretenden Bürgermeisters, sowie die Entwicklung eines Amtes für Denkmalschutz und Architektur. Der Artikel beleuchtet die Art und Weise, wie Fragen des Denkmalschutzes öffentlich behandelt werden. Vom Standpunkt der jeweiligen Berufskulturen aus werden Konfrontationen und Artikulationen zwischen unterschiedlichen Sichtweisen und Know-hows des neuen Amtes für Denkmalschutz und der Ämter für Stadtplanung untersucht. In Verbindung mit anderen Akteuren (Historiker, Vereine, touristische Strukturen) betreiben diese Ämter Städtebau, sowohl in langzeitlicher Hinsicht (Stadtprojekt mit sich entwickelnden Zielen) als auch im Rahmen von der Austeilung von Baugenehmigungen. Hier zeigen sich die komplexen Beziehungen zwischen Kulturerbe und Urbanismus, die ein Nachdenken nötig machen über die Wertesysteme im zeitgenössischen Raum. Dienstanweisungen (Expertisen), Vorschriften (Regeln) und konkrete Operationen (Baustellen) zeugen von einem entscheidenden Entwicklungsmoment der Beziehungen zwischen den betroffenen öffentlichen Ämtern.

\section{AUTHORS}

\section{LAURENT DEVISME}

LAUA, Ensa Nantes

\section{AMÉLIE NICOLAS}

LAUA, Ensa Nantes 